\title{
Sistema de recomendação baseado no modelo de reconhecimento de atividades HARA
}

\author{
Gabriel Di iorio Silva, Victor Ströele, Mario Dantas \\ ${ }^{1}$ Departamento de Ciência da Computação - Universidade Federal de Juiz de Fora (UFJF) \\ \{iorio,victor.stroele, mario.dantas\}eice.ufjf.br
}

\begin{abstract}
Resumo. Formas de se monitorar continuamente a saúde humana vem se tornando populares, principalmente devido ao alargamento de nossa pirâmide etária. Nesse cenário ambientes assistidos surgem como uma tecnologia para atender às necessidades desse público alvo. Técnicas que detectam as atividades realizadas por um indivíduo se mostram promissoras para esse problema uma uma vez que ao se identificar as ações podemos perceber anomalias na rotina de um usuário que podem sinalizar estágios iniciais de uma doença. Sistemas de recomendação surgem como uma maneira de agregar funcionalidades à ambientes assistidos com o intuito de realizar recomendações para o usuário final ou o especialista médico de acordo com a gravidade notada da anomalia. Estudos iniciais mostram que a proposta de mesclar sistemas de recomendação com ambientes assistidos é viável e diferencial dado que essa junção é pouco explorada.
\end{abstract}

\section{Introdução}

Segundo pesquisas realizadas em 2016 pelo IBGE, o número de idosos no Brasil triplicará em 40 anos e passará de $10 \%$ da população brasileira, em 2010, para 29,3\% em 2050 [IBGE 2016]. Com o advento da internet e tecnologias móveis cada vez mais presentes em nosso cotidiano, termos como computação ubíqua foram utilizados para designar a onipresença da informática em nossas rotinas. Somando isso ao cenário atual de envelhecimento da população brasileira, tecnologias que permitam uma melhor qualidade de vida e que possam auxiliar na saúde tornam-se promissoras, permitindo monitorar pessoas em seus ambientes domiciliares, os chamados ambientes domiciliares assistidos.

Em geral, essas abordagens utilizam sensores para monitorar os usuários. Neste trabalho, para interpretar os dados recolhidos dos acelerômetros, foi utilizado o modelo HARA (Human Activity Recognition with Accelerometer) [Amaral and Dantas 2017], que consiste no armazenamento dos dados de posicionamento na residência, movimentação e tempo, sendo possível inferir as atividades diárias de um indivíduo, e assim entender sua rotina. Portanto, baseando-se nos dados coletados pelos acelerômetros e interpretados pelo modelo HARA, seremos capazes de recomendar ações, como a sugestão de atividades para redução de sedentarismo, e, até mesmo, sugerir que o usuário procure um especialista da área de saúde, aumentando as chances de um tratamento bem-sucedido, pela detecção precoce de uma doença.

Os Sistemas de Recomendação (SR) buscam apresentar informações relevantes e personalizadas para os usuários considerando suas necessidades. No contexto da saúde, esses sistemas podem ser utilizados para recomendar conteúdos relevantes para 
a saúde dos usuários. Os sistemas de recomendação também podem ser utilizados no ramo acadêmico para recomendar artigos, trabalhos e vídeos de acordo com a necessidade, ramo de pesquisa e objetivo do usuário. Além disso, podemos observar sistemas de recomendação voltados para o lazer, seja na recomendação de músicas ou filmes de acordo com os gostos interpretados e analisados, tornando assim sua experiência mais agradável e prática na plataforma.

Portanto, dado esse contexto e a ampla utilização dos sistemas de recomendação, o objetivo desse trabalho é desenvolver um SR que atue conforme as atividades identificadas pelos acelerômetros e interpretadas pelo modelo HARA, consiga enviar relatórios para especialistas médicos, além de recomendar devidas ações para usuários que apresentarem atividades anômalas para prevenir possíveis agravações de doenças, como também melhorar a qualidade de vida dos usuários.

Este trabalho está organizado como segue: na Seção 2 é apresentada a fundamentação teórica, na Seção 3 segue a motivação, na Seção 4 são apresentados os trabalhos correlatos, Na seção 5 arquitetura do modelo HARA-RS; na Seção 6 uma avaliação preliminar é conduzida e, na Seção 7, são feitas as considerações finais.

\section{Fundamentação Teórica}

Nessa seção são apresentados os pressupostos teóricos que dão o embasamento necessário para a compreensão do problema e da proposta apresentada. Os conceitos relacionados a sistemas de recomendação e suas técnicas de filtragem, métodos e afins são de suma importância para elucidar os termos que serão comentados nesse trabalho.

\subsection{Sistema de Recomendação}

Segundo [Burke 2002], sistema de recomendação é: "qualquer sistema que produza recomendações individualizadas ou que tenha o efeito de guiar o usuário de forma personalizada para objetos do seu interesse ou que lhes sejam úteis dentre diversas opções possíveis". Esse sistema precisa, fundamentalmente, de três artifícios para que possa ter pleno funcionamento. (1) Os dados de perfil, que são informações que o sistema possui antes de fornecer a recomendação. (2) Os dados de entrada, que são informações coletadas sobre o usuário com o propósito de se realizar uma recomendação, e (3) o algoritmo do sistema de recomendação no qual está presente as técnicas e formas com que o sistema manipula os dados identificados e, a partir deles, realiza a melhor recomendação.

\subsection{Técnicas de Recomendação}

Para que haja a recomendação, metodologias são utilizadas no o algoritmo com o propósito de melhorar o conteúdo recomendado para que esse se adéque as peculiaridades do usuário, tais como: filtragem, perfil e contexto. Nessa subseção serão apresentados os conceitos a respeito dos artifícios empregados na camada de recomendação.

Nas mais diversas aplicações de um sistema de recomendação, percebemos diferentes formas de se abordar um problema; temos como exemplo: filtragem colaborativa, filtragem baseada em conteúdo, filtragem social e a filtragem híbrida. Dentre elas vamos ressaltar a filtragem demográfica, a filtragem Baseada em Conteúdo e a filtragem híbrida. A filtragem demográfica faz o uso de dados coletados acerca de um determinado grupo no qual notamos seus gostos e preferências para que assim, conforme o enquadramento 
de um usuário em certo grupo demográfico, o sistema é guiado para que atenda àquelas inclinações [J. Bobadilla ]. A filtragem baseada em conteúdo compara o quão similar são as possíveis recomendações com o perfil do usuário, para que assim o mesmo receba apenas conteúdos relacionados a seus gostos. Por fim, a filtragem híbrida é aquela que mistura dois ou mais tipos diferentes de filtragem. O sistema proposto neste trabalho utiliza a filtragem híbrida, mesclando a filtragem demográfica e a filtragem baseada em conteúdo[?].

\subsection{Perfil e Contexto}

Para realizar a recomendação, é necessário notar o perfil e contexto do usuário que irá receber a recomendação. Nesse sentido são usados dados extraídos ou inseridos no sistema. O perfil, em nosso caso, pode ser definido como explícito, uma vez que depende do usuário para que o mesmo adicione informações predefinidas e úteis, para que haja o pleno funcionamento do sistema. Sob a perspectiva do contexto, podemos defini-lo seguindo a noção de [Fle ], que o estabelece como qualquer informação inserida ou capturada pelo sistema que influencie na sua usabilidade. Assim, nosso contexto provém das atividades que o usuário está realizando, dada a ação reconhecida pelo sistema HARA-RS, esse será seu contexto atual.

\subsection{Ambientes Assistidos}

Um Ambiente Domiciliar Assistido consiste em um conjunto heterogêneo de sensores corporais e de ambiente que dão origem a um grande volume de dados[Abdur Forkan 2014]. Portando, logramos um ambiente delimitado que verifica as atividades e coleta dados acerca da rotina de um indivíduo para gerar informações sobre sua saúde. Tais sensores podem prover dados de localização, movimento ou ambos. Também podemos varias a posição dos sensores corporais de forma a obter dados mais precisos. Em nosso sistema HARA-RS mesclamos dados de localização com dados de movimento para informações aprimoradas.

\section{Motivação}

Dado o contexto do envelhecimento da população mundial, comentado na seção 1, tecnologias de monitoramento constante da saúde se tornaram ainda mais presentes em nosso cotidiano, como: Xiaomi mi band e Smartband. Sua disseminação se deve principalmente ao fato de terem baixo custo, são pouco intrusivas e o fato da informática ser intrínseca às atividades da população. Ambientes assistidos despontam como uma forma não intrusiva, conveniente e confortável para que um usuário tenha sua saúde monitorada de forma constante.

Sistemas de recomendação, quando atrelados à ambientes assistidos se mostram extremamente eficientes e úteis. Aliando a premissa de se realizar recomendações de conteúdos interessantes seguindo suas preferências, perfil e contexto identificados. Assim, ampliamos as funcionalidades de um ambiente assistido, que coleta informações à respeito de uma pessoa, para que o mesmo seja capaz de prover as melhores recomendações médicas conforme os dados identificados.

Tais recursos, entretanto, mostram-se estáticos em virtude de não serem sistemas livres e open source, ou seja, não possuem seus códigos abertos de forma que possa ser 
adaptado para diferentes fins e utilizados gratuitamente. Tal lacuna revela-se interessante para progressão de pesquisas acadêmicas com esse cunho. Agregando o fato supracitado à pouca exploração de técnicas que mesclam ambientes assistidos com sistemas de recomendação, o trabalho emerge como uma forma de agregar ferramentas para que as mesmas sejam aplicadas nos mais diferentes ramos do conhecimento.

\section{Trabalhos Correlatos}

Ultimamente, muitos trabalhos passaram a abordar o tema de SR voltados para a saúde. Por se tratar de um tema relativamente novo e levando em consideração o cenário futuro, com a expectativa de vida se elevando cada vez. Dessa forma, alguns trabalhos como [Wiesner and Pfeifer 2014], apresentam sistemas que, com base no perfil do usuário, recomendam artigos e textos que ajudam a entender seu estado clínico ou até mesmo recomendar textos que abordam temas na saúde, porém com uma linguagem de fácil entendimento de acordo com o usuário.

Outros trabalhos utilizam sensores para analisar a saúde do usuário e coletar dados que são enviados para um especialista ou para a análise por parte do próprio indivíduo [Amay J Bandodkar 2014]. Entretanto, são poucos trabalhos que focam em soluções que utilizam sensores com o intuito de coletar e transmitir dados para o usuário final. [Lee and Chung 2009] propuseram o uso de camisas inteligentes, compostas por alguns sensores, que coletam os dados do indivíduo por meio de acelerômetros. Tal sistema é baseado em uma arquitetura na qual os dados coletados são enviados para um repositório único com todas as informações obtidas pelos sensores. Outros sistemas utilizam variados tipos de sensores, capazes de enviar os dados coletados para uma pessoa especializada ou para que o próprio usuário realize a análise dos dados coletados, tendo arquiteturas variadas de acordo com a necessidade do usuário [Jung et al. 2008].

Ademais, sensores com propostas diferentes relacionados com a extração de dados podem ser observados, como o trabalho supracitado, que faz uso de sensores eletroquímicos [Amay J Bandodkar 2014] que objetivam monitorar o estado metabólico, hormonal e emocional do indivíduo. A coleta de dados realiza uma comparação com os níveis normais para aquela determinada parte analisada, como o nível do PH encontrado na saliva e o nível normal para um ser humano.

Ainda temos, juntamente, trabalhos que abordam a arquitetura por trás de um ambiente assistido, como evidenciado em [Chris Otto 2006]. Tal arquitetura é bastante semelhante a usada em nossa proposta em que os sensores coletam os dados. Posteriormente os mesmos são distribuídos para a camada que contém o dispositivo pessoal do usuário no qual os dados são enviados via Bluetooth ou conexão ZigBee. Subsequente, os dados são enviados para uma base médica via $2 \mathrm{G}$ ou $3 \mathrm{G}$ para que assim possam ser revisados por médicos.

Para visualizar algumas peculiaridades e características do sistema HARA-RS em relação aos trabalhos relacionados acima temos a tabela comparativa a seguir.

O uso de sensores é amplamente difundido em pesquisas científicas, pois, dessa forma, o sistema se torna ainda mais personalizado para o usuário. Neste sentido, este trabalho utiliza os sensores de forma a criar um ambiente assistido e realizar o reconhecimento das atividades dos usuários, além de enviar a devida recomendação de acordo com 
Tabela 1. Tabela comparativa

\begin{tabular}{|c|c|c|c|c|c|}
\hline & HARA-RS & Amay & Wiesner & Young & Otto \\
\hline \multicolumn{6}{|l|}{ Sensores Corporais } \\
\hline \multicolumn{6}{|l|}{ Sensores de Ambiente } \\
\hline \multicolumn{6}{|l|}{ Sensores Eletroquímicos } \\
\hline \multicolumn{6}{|l|}{$\begin{array}{l}\text { Sistema de } \\
\text { Recomendação }\end{array}$} \\
\hline \multicolumn{6}{|l|}{ Não é intrusivo } \\
\hline $\begin{array}{l}\text { Funciona em qualquer } \\
\text { locação }\end{array}$ & & & & & \\
\hline
\end{tabular}

a interpretação das atividades reconhecidas pelo sistema. Diferencia-se essa pesquisa de outros trabalhos relacionados, que monitoram atividades como o Apple Watch e Jawbone Pulseira UP, posto que efetua-se recomendação de acordo com o contexto identificado.

\section{HARA-RS: Recommender System based on Human Activity Recognition with Accelerometer}

Este trabalho propõe o HARA-RS, um Sistema de Recomendação baseado nos dados obtidos e analisados do modelo HARA para que, por meio das atividades realizadas por um usuário monitorado em casa, o sistema seja capaz de recomendar, através das anomalias detectadas pelo modelo, que o indivíduo se dirija a um hospital próximo, que seu tempo de sono está irregular, problemas relacionadas ao sistema gastrointestinal, sedentarismo, entre outros tipos de recomendação. Escolheu-se o modelo HARA para o monitoramento e interpretação das atividades em razão de que o mesmo foi desenvolvido pelo nosso grupo de pesquisa.

A Figura 2 apresenta a arquitetura em camadas do HARA-RS. Os dados de atividades dos usuários são coletados e transmitidos, por Bluetooth, para o modelo HARA. Esses dados são interpretados de modo a definir o perfil e o contexto do usuário, que são usados pelo sistema de recomendação para apresentar conteúdos personalizados ao mesmo. Cada uma das camadas dessa arquitetura são descritas detalhadamente nas próximas subseções.

\subsection{Camada de Coleta de dados}

Esta camada é responsável por coletar os dados dos usuários que irão receber recomendações. A coleta dos dados é feita por meio de dispositivos bluetooth espalhados 


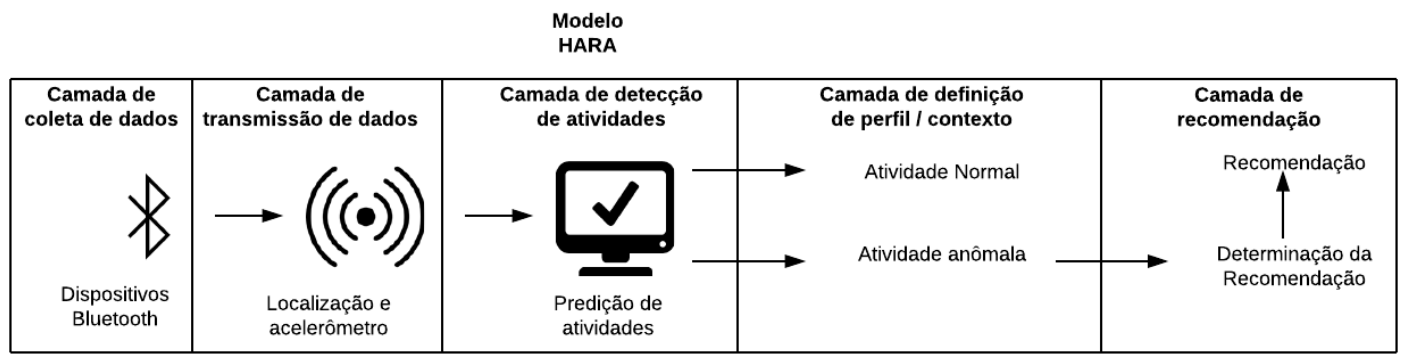

Figura 1. Sistema de recomendação - Visão geral

pela casa (ambiente assistido). Portanto, é possível mapear a intensidade do sinal enviado pelo sensor corporal e definir em qual cômodo a pessoa está. Neste artigo foram utilizados os dados já coletados previamente no experimento de [Amaral and Dantas 2017].

\subsection{Cama de Transmissão}

Esta camada realiza a transmissão dos dados de movimentação e localização coletados, os mesmos foram transmitidos para o microcontrolador e computador (Raspberry Pi 3) para que assim fossem interpretados pelo modelo HARA-RS.

\subsection{Camada de Detecção das Atividades}

Esta camada faz a interpretação dos dados coletados, com o intuito de classificar os cômodos da casa e reconhecer as atividades realizadas pelos usuários. Para identificar a localização do indivíduo na casa, dispositivos Bluetooth foram espalhados pelo ambiente. Tais dispositivos são responsáveis por verificar a intensidade do sinal em relação a distância do dispositivo com o acelerômetro usado no corpo do usuário. Nessa etapa, foi necessário configurar o sistema manualmente para que fique salvo em uma base de dados uma relação entre intensidade de sinal e cômodo da casa, para que assim possamos relacionar tais dados e fazermos as recomendações devidas.

Para o reconhecimento das atividades dos usuários, a técnica utilizada pelo modelo HARA é o Modelo Oculto de Markov (HMM), seguindo sua arquitetura. A escolha pelo HMM se deve ao fato de ser possível realizar infinitas sequências com uma quantidade finita de termos. Analogamente, teremos uma sequência infinita de ações a partir de uma quantidade finita de estados. Ademais, é implementado um modelo que avalie a qualidade de contexto para decidir se os dados coletados devem ser descartados ou devem gerar um alerta.

\subsection{Camada de Definição do Perfil e Contexto}

Esta camada realiza a definição do perfil do usuário com base em dados previamente especificados como idade, sexo, peso, problemas de saúde, dentre outras características. Conjuntamente com os dados que foram interpretados pelo modelo HARA, o sistema define o contexto em que o usuário se encontra; que atividades ele realizou e se houveram atividades anômalas encontradas de acordo com sua rotina e com seu perfil, para assim estabelecer as possíveis recomendações para esse usuário. 
O sistema de recomendação irá classificar a atividade a partir da interpretação dos dados, realizada pelo modelo HARA. Seguindo a devida classificação o sistema é capaz de reconhecer a atividade anômala e fazer a recomendação adequada. A partir do momento em que tais atividades anômalas se tornam corriqueiras, detectadas diversas vezes na semana ou no dia, o sistema irá detectar tal comportamento e efetuar um outro tipo de recomendação, dada a possível gravidade ou risco desses comportamentos. Logo, o sistema irá realizar uma filtragem baseada em conteúdo, definindo o perfil e o contexto de acordo com a atividade detectada.

\subsection{Camada de Recomendação}

Nesta camada é executado um Sistema de Recomendação considerando diferentes instanciações da arquitetura HARA-RS. A proposta deste trabalho é possibilitar que o sistema seja utilizado por diferentes demandas do domínio de saúde. Basicamente, os usuários finais, que receberão as recomendações podem ser classificados em: especialistas da área e pessoas com necessidade ou interesse em algum tipo acompanhamento.

Independentemente do usuário final, todas as outras camadas são utilizadas da mesma forma, apenas a camada de recomendação é configurada para filtragem dos conteúdos adequados ao usuário que receberá a recomendação. Essa configuração evita que conteúdos destinados a especialistas sejam recebidos por usuários comuns. Alguns exemplos de uso da arquitetura e sua visão geral são descritos a seguir.

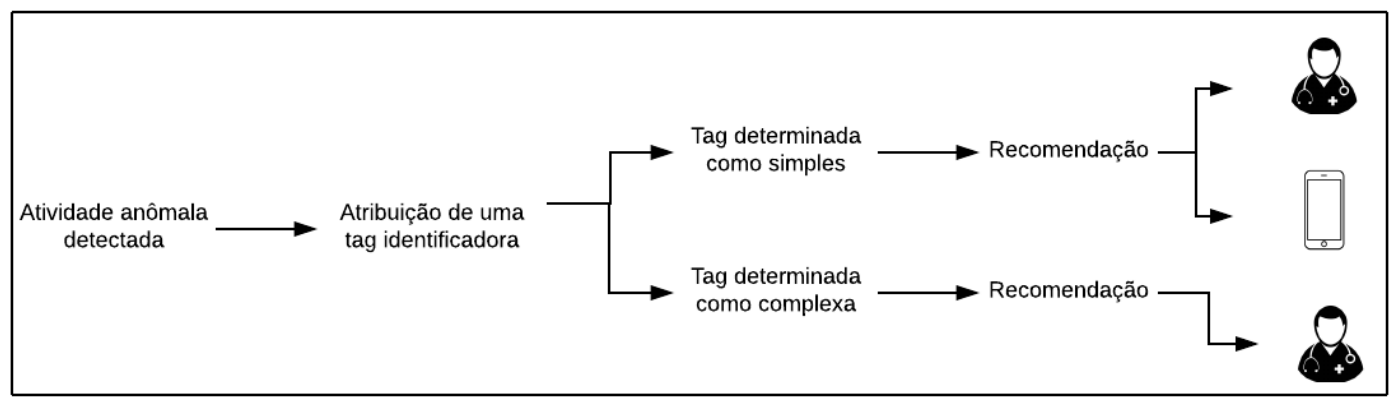

Figura 2. Camada de recomendação - Visão geral

A arquitetura pode ser instanciada considerando que quem recebe a recomendação é um usuário comum sem a presença de especialista médico. Nesse caso a recomendação é feita de forma a indicar para o usuário soluções simples e diretas, levando-se em consideração a falta de um especialista para recomendações que não cabem a um sistema sem suporte de um profissional médico.

Outra forma de instanciação pode ser considerada quando tratamos de um usuário comum com um especialista médico. Assim, a recomendação é feita de forma a distinguir quais recomendações são feitas para o profissional e quais são destinadas ao usuário comum. Portanto, o especialista é capaz de, com base nas informações e recomendações recebidas, determinar qual a melhor forma de tratamento ou prevenção para aquele usuário.

Uma terceira instanciação pode ser verificada ao tratarmos de um usuário menor de idade ou idoso com necessidades especiais. Diante disso, a recomendação é feita de 
forma a enviar os dados de recomendação para o indivíduo responsável pelo usuário, uma vez que podemos tratar de crianças ou idosos que não possuem condições de receberem tais recomendações indicadas pelo sistema e realizar o que o sistema sugere.

Ademais, podemos tratar de outros casos nos quais usuários comuns têm o interesse de serem monitorados para algum propósito. Podemos considerar, por exemplo, um usuário que deseja acompanhar sua queima de calorias. Nesse cenário, a recomendação é feita com base na quantidade de calorias que o usuário deseja perder por dia, definindo assim mais atividades para serem realizadas caso não se alcance a meta esperada, de modo a auxiliar o mesmo em seu objetivo.

Dadas as especificações do sistema, podemos classificá-lo como sendo um sistema sensível ao contexto, em virtude de utilizar o contexto, ou seja, a atividade que o usuário está realizando em determinado momento, para que sejam feitas as recomendações. Outrossim, também podemos atribuí-lo a característica de um sistema baseado em conteúdo porquanto utiliza tags descritoras de itens. Conforme a atividade detectada lhe é atribuída uma tag e consoante com as tags em nossa base de dados, iremos realizar a melhor recomendação por meio do matching entre as mesmas. [de Souza 2014].

Neste sentido, o sistema faz uso de uma filtragem híbrida que mescla a filtragem baseada em conteúdo com a filtragem demográfica. É interessante fazer uso das funcionalidades da filtragem demográfica em razão de algumas doenças serem mais recorrentes em usuários de determinado sexo ou com certa idade, peso e histórico de doenças crônicas. Assim o sistema melhora sua precisão fazendo com que suas recomendações fiquem ainda mais exatas.

Durante a recomendação devemos ser capazes de identificar os limites entre a interação do sistema de recomendação e o usuário para que o mesmo não substitua o profissional médico. Dessarte, o HARA-RS visa ser uma forma de estender e auxiliar o especialista. Tendo isso em vista, conforme as possíveis tags que o sistema é capaz de atribuir à certas atividades, um profissional médico atribuiu à todas as tags, durante a configuração do sistema e do algoritmo, um grau de risco. Em concordância com a atividade detectada e o grau a ela dado, se esse grau ultrapassa o limitante definido como casos pequenos e de fácil resolução, as devidas recomendações serão então enviadas somente para o médico, e, ao usuário, é enviado uma mensagem alertando-o sobre esse fato. Todavia, se tal tag está abaixo do limitante, a recomendação é enfim enviada a ambos, usuário e médico.

\section{Avaliação preliminar}

Para avaliar o modelo HARA-RS, foi conduzido um experimento preliminar, instanciando a arquitetura considerando um usuário que deseja monitorar seu gasto diário de calorias. Os dados foram monitorados, transmitidos e as atividades foram detectadas através do modelo HARA. Com base nessas atividades e nas informações dos usuários, o sistema identifica o perfil e o contexto do mesmo. As características de perfil identificadas foram: usuário do sexo masculino, idade entre 20 e 30 anos, que não possui, declaradamente, nenhum problema de saúde, e tem por objetivo gastar $1000 \mathrm{kcal}$ por dia. O contexto foi definido através do monitoramento e consolidação das atividades diárias do usuário. A Figura ?? lista as atividades identificadas e os seus respectivos gastos calóricos ${ }^{1}$, $\operatorname{compondo}$

\footnotetext{
${ }^{1}$ www.bemestar.globo.com
} 
o contexto do usuário.

Tabela 2. Tabela de atividades e gastos energéticos

\begin{tabular}{|cc|}
\hline Atividade & Calorias gastas \\
Cozinhar & $168 \mathrm{kcal} /$ hora \\
Dormir & $77 \mathrm{kcal} / \mathrm{hora}$ \\
Andar & $5,5 \mathrm{kcal} / \mathrm{min}$ \\
Lavar louça & $60 \mathrm{kcal} / \mathrm{hora}$ \\
Escrever & 10 a $20 \mathrm{kcal} / \mathrm{hora}$ \\
Usar computador & $95 \mathrm{kcal} / \mathrm{hora}$ \\
Escovar os dentes & $80 \mathrm{kcal} / \mathrm{hora}$ \\
Comer & $105 \mathrm{kcal} / \mathrm{hora}$ \\
Arrumar a cama & $132 \mathrm{kcal} / \mathrm{hora}$ \\
Varrer & $214 \mathrm{kcal} / \mathrm{hora}$ \\
\hline
\end{tabular}

Portanto, de acordo com a Figura 3, que ilustra a instanciação desse modelo, os dados de movimentação e localização foram coletados pelos dispositivos bluetooth espalhados pelo ambiente assistido e pelos acelerômetros. Logo em seguida foram transmitidos para a camada de detecção de atividades que reconheceu e interpretou essas atividades e então, esses dados foram enviados para a camada de recomendação. Com os dados coletados nesse experimento, o perfil e o contexto do usuário foi consolidado, considerando um dia de atividades: Dormir(2 horas), Arrumar a cama(20 minutos), Lavar louça(1 hora), Escovar os dentes(10 minutos), Usar o computador(3 horas), Cozinhar(1 hora).

Foi identificado que o usuário gastou, considerando seu contexto, aproximadamente $676,33 \mathrm{kcal}$. Desta forma, a camada de recomendação sugeriu que o usuário praticasse alguma atividade leve para que ele alcançasse o seu objetivo de gasto calórico.As recomendações foram enviadas somente para o usuário final uma vez que o mesmo não possui especialista médico.

\section{Considerações Finais}

Este trabalho apresentou o modelo HARA-RS, capaz de identificar as atividades realizadas por pessoas em um ambiente assistido, recomendando ações para essas pessoas. Uma avaliação preliminar foi conduzida mostrando a viabilidade do modelo em indicar para o usuário medidas a serem tomadas conforme as atividades detectadas. O sistema também aborda a ideia de várias instanciações da arquitetura para atender diversas demandas de acordo com as possíveis necessidades até agora previstas. Como trabalhos futuros 


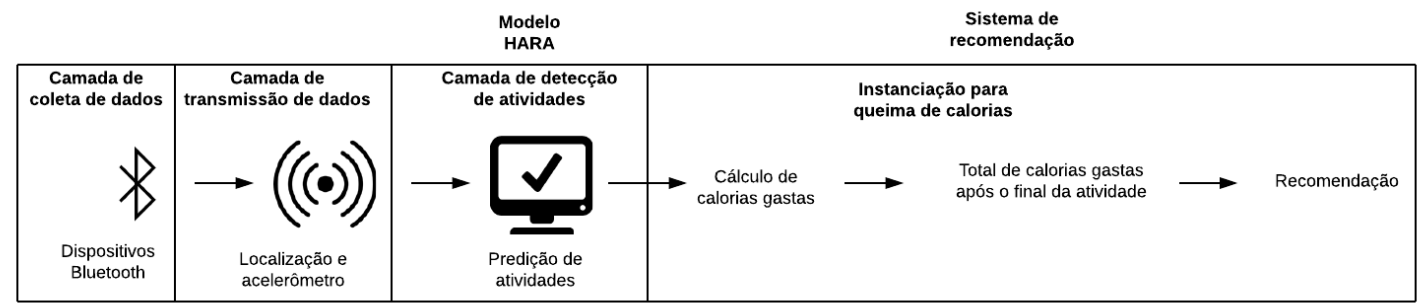

Figura 3. Instanciação para gasto de calorias - Visão geral

pretende-se avaliar o sistema em outros ambientes assistidos (domiciliar ou hospitalar) e com as outras instâncias monitorando usuários com interesse em receber recomendações de conteúdo personalizado relacionados a saúde. Ademais é interessante definir uma base de dados de extrema confiança para que não haja dúvidas acerca do que está sendo recomendado.

\section{Referências}

Abdur Forkan, Ibrahim Khalil, Z. T. S. F. A. B. (2014). A context-aware approach for long-term behavioural change detection and abnormality prediction in ambient assisted living.

Amaral, W. and Dantas, M. (2017). Um modelo de reconhecimento de atividades humanas baseado no uso de acelerometro com qoc. In Workshop de Iniciação Científica em Arquitetura de Computadores e Computação de Alto Desempenho (WSCAD-WIC 2017), pages 45-50. Porto Alegre: SBC.

Amay J Bandodkar, J. W. (2014). Non-invasive wearable electrochemical sensors: A review. Trends in Biotechnology, 32(7):363-371.

Burke, R. (2002). Hybrid recommender systems: Survey and experiments.

Chris Otto, Aleksandar Milekovic, C. S. E. J. (2006). System architecture of a wireless body area sensor network for ubiquitous health monitoring.

de Souza, R. G. D. (2014). Sistemas de recomendação aplicando sistemas de recomendação em situações práticas.

IBGE (2016). Projeção da população do brasil. Revisão 2016.

J. Bobadilla, F. Ortega, A. H. A. G. Knowledge-based systems.

Jung, J., Ha, K., Kim, J. L. Y., and Kim, D. (2008). Wireless body area network in a ubiquitous healthcare system for physiological signal monitoring and health consulting. International Journal of Signal Processing, Image Processing and Pattern Recognition.

Lee, Y.-D. and Chung, W.-Y. (2009). Wireless sensor network based wearable smart shirt for ubiquitous health and activity monitoring. Sensors and Actuators B: Chemical, 140(2):390-395. https://doi.org/10.1016/j.snb.2009.04.040. 
Wiesner, M. and Pfeifer, D. (2014). Health recommender systems: Concepts, requirements, technical basics and challenges. International Journal of Environmental Research and Public Health (IJERPH), 11(3):2580-2607. https://doi.org/10.3390/ijerph110302580. 